Article

\title{
Probabilistic Interval-Valued Hesitant Fuzzy Information Aggregation Operators and Their Application to Multi-Attribute Decision Making
}

\author{
Wenying $\mathrm{Wu}^{1,2}\left(\mathbb{D}\right.$, Ying $\mathrm{Li}^{1,2, *}$, Zhiwei $\mathrm{Ni}^{1,2}$, Feifei Jin ${ }^{1,2}$ and Xuhui Zhu ${ }^{1,2}$ \\ 1 School of Management, Hefei University of Technology, Hefei 230009, China; \\ hfut.wwying@gmail.com (W.W.); nzwgd@hfut.edu.cn (Z.N.); feifeijin88@gmail.com (F.J.); \\ hfutzxh@gmail.com (X.Z.) \\ 2 Key Laboratory of Process Optimization and Intelligent Decision-Making, Ministry of Education, \\ Hefei 230009, China \\ * Correspondence: hgdly@126.com; Tel.: +86-139-5606-6316
}

Received: 9 July 2018; Accepted: 28 July 2018; Published: 6 August 2018

\begin{abstract}
Based on the probabilistic interval-valued hesitant fuzzy information aggregation operators, this paper investigates a novel multi-attribute group decision making (MAGDM) model to address the serious loss of information in a hesitant fuzzy information environment. Firstly, the definition of probabilistic interval-valued hesitant fuzzy set will be introduced, and then, using Archimedean norm, some new probabilistic interval-valued hesitant fuzzy operations are defined. Secondly, based on these operations, the generalized probabilistic interval-valued hesitant fuzzy ordered weighted averaging (GPIVHFOWA) operator, and the generalized probabilistic interval-valued hesitant fuzzy ordered weighted geometric (GPIVHFOWG) operator are proposed, and their desirable properties are discussed. We further study their common forms and analyze the relationship among these proposed operators. Finally, a new probabilistic interval-valued hesitant fuzzy MAGDM model is constructed, and the feasibility and effectiveness of the proposed model are verified by using an example of supplier selection.
\end{abstract}

Keywords: multi-attribute group decision making; probabilistic interval-valued hesitant fuzzy sets; Archimedean norm; information aggregation operators; supplier selection

\section{Introduction}

Since Zadeh [1] put forward the theory of the fuzzy set (FSs), the decision making problem of fuzziness and uncertainty existing in real life has been dealt with better. Then, several extensions of fuzzy sets were proposed. On the basis of membership degree, the membership degree and hesitant degree were added, and the concept of intuitionistic fuzzy sets (IFSs) was introduced by Atanassov [2], which is used to process decision information. Torra [3] proposed the concept of hesitant fuzzy set, which effectively solves the hesitation of experts in group decision making and is better used to express decision information.

In real life, many decision making problems are difficult to represented by an accurate value, and a relatively reasonable interval-value is chosen to describe them. On the basis of intuitionistic fuzzy sets, Atanassov [4] introduced interval-valued concept, and put forward interval-valued intuitionistic fuzzy sets to solve decision problems. With the concept of interval-valued intuitionistic fuzzy sets, some scholars have studied the basic operations and basic properties of interval-valued intuitionistic fuzzy sets. In the context of interval-valued intuitionistic fuzzy sets, Garg [5] defined a new score function by adding weighted average of hesitant degree in membership function to deal 
with the decision process. Subsequently, Zhang [6] reviewed the literature [5] and commented on the shortcomings of Garg's paper. Under interval-valued intuitionistic fuzzy sets, entropy measures have been studied by different researchers, which can describe the uncertainty information more fully [7-10]. Chen [11] proposed a new multi attribute decision making (MADM) method based on the interval-valued intuitionistic fuzzy weighted geometric average (IIFWGA) operator of interval-valued intuitionistic fuzzy values (IVIFVs), the accuracy function of IVIFVs and Particle Swarm Optimization techniques, which can overcome the drawbacks of the existing MADM methods for MADM in interval-valued intuitionistic fuzzy (IVIF) environments. The decision making process of actual problems has certain uncertainty. In order to depict the uncertainty in decision process more effectively, a new interval-valued hesitant fuzzy sets is defined by Chen [12], which is based on the properties of hesitant fuzzy sets and the situation where the evaluation values are interval values. Under the interval-valued hesitant fuzzy sets, based on the continuous ordered weighted averaging (COWA) operator, Jin [13] constructed several information measure formulas for interval-valued hesitant fuzzy elements (IVHFEs) and discussed the relationship among these information measures for IVHFEs. Liu [14] studied the multi attribute group decision making (MAGDM) method which combines the improved Hamacher aggregation operators and continuous entropy. To address the lack of flexibility in decision making and lack of original information, Lan [15] proposed some priority formulas of interval-valued hesitant fuzzy sets. Simultaneously, the expectation property of priority degree is also studied. In the multi attribute group decision making analysis of interval valued intuitionistic fuzzy information, the determination of attribute weight has a direct impact on the choice of the optimal scheme [16]. In view of complex group decision making problem, a multi-criteria weighting and ranking model is introduced with interval-valued hesitant fuzzy sets by Gitinavard [17], which operates properly under uncertainties. Furthermore, the attribute weights of interval numbers are discussed by both Shang [18] and Li [19] to solve the MAGDM problem. Jin et al. [20] constructed a new group decision making model for interval-valued hesitant fuzzy MAGDM problems with priority relations among attributes.

The uncertainty of individual decision making is an important factor that ultimately affects the consistency of group decision making. Although hesitant fuzzy sets can better express the ideas of experts, there is the problem of information loss, and it cannot fully reflect the decision information. To cope with this issue, some researchers have introduced the concept of probability. For example, Hang and $\mathrm{Xu}$ [21] introduced probability into hesitant fuzzy sets, and put forward the concept of probabilistic hesitant fuzzy sets, and gave its operation rules. Wang [22] introduced a MAGDM method based on correlation coefficient to use probabilistic hesitant fuzzy information. It is undeniable that researchers have effectively improved and extended the hesitant fuzzy sets to make it more comprehensive to express decision information. Zhai et al. [23] defined a probabilistic interval-valued intuitionistic hesitant fuzzy set (PIVIHFS) as an extended mathematical expression of fuzzy sets and established some new measure models deduced by the axiomatic concepts of PIVIHFSs. Liu [24] proposed the probabilistic linguistic term sets (PLTSs), in which each provided linguistic term has a probability. Farhadinia [25] defined the modified-PLTS (M-PLTS) and transformed it to the ordered weighted hesitant fuzzy element (OWHFE). Li [26] built a consensus among the decision makers for probabilistic hesitant fuzzy preference relations (PHFPRs) with expected additive consistency. Zhou [27] proposed the uncertain probabilistic hesitant fuzzy element (UPHFE) and extended it to the uncertain probabilistic hesitant fuzzy preference relations (UPHFPRs).

On the one hand, the aggregation of decision attribute information is an important research topic in MAGDM problems. From the above analysis, we can find that the most of current interval-valued information aggregation operators are put forward under interval-valued hesitant fuzzy information and interval-valued intuitionistic fuzzy information environment. However, there is no research on the probabilistic interval-valued hesitant fuzzy information aggregation operators at present. On the other hand, although there exist some useful algorithms, there are still some problems such as loss of decision information and inaccuracy of decision results. In this paper, the concept of probability is added to give 
the corresponding degree of possibility for decision information value, which enhances the reliability and effectiveness of decision results. In addition, the Archimedes norm makes the aggregation algorithm more flexible and the decision making process more convenient. Therefore, the algorithm model proposed in this paper is necessary.

In this paper, we apply the Archimedes norm to investigate the aggregation method for probabilistic interval-valued hesitant fuzzy information. To do so, the rest of this paper is structured as follows. In Section 2, we review some basic concepts and notations of the IVHFSs and define the PIVHFSs. Then, we introduce the Archimedes norm, and (based on this) the probabilistic interval-valued hesitant fuzzy operation rules are discussed. In Section 3, we define the probabilistic interval-valued hesitant fuzzy information aggregation operators whose related properties are explored in detail. Based on the additive operator $g(t)$ which takes different functions, Section 4 studies some common information aggregation operators and analyzes the relationship among these proposed operators. Based on the proposed operators, a method to multiple attribute group decision making with probabilistic interval-valued hesitant fuzzy information is provided in Section 5. An example is given to show the effectiveness and feasibility of the proposed method in Section 6. Section 7 summarizes the full text and draws the main conclusions.

\section{Preliminaries}

\subsection{Interval-Valued Hesitant Fuzzy Sets}

Definition 1. Refs. [28,29]. Let $X=\left\{x_{1}, x_{2}, \cdots, x_{n}\right\}$ be a given set, and $D[0,1]$ be the set of all closed subintervals of $[0,1]$. An IVHFS on $\mathrm{X}$ is

$$
\widetilde{A}=\left\{\left\langle x_{i}, \widetilde{h}_{\widetilde{A}}\left(x_{i}\right)\right\rangle \mid x_{i} \in X\right\},
$$

where $\widetilde{h}_{A}\left(x_{i}\right): X \rightarrow D[0,1]$ denotes all possible interval-valued membership degrees of the element $x_{i} \in X$ to the set $\widetilde{A}$. For convenience, we call

$$
\widetilde{h}_{\widetilde{A}}\left(x_{i}\right)=\left\{\widetilde{\gamma} \mid \widetilde{\gamma} \in \widetilde{h}_{\widetilde{A}}\left(x_{i}\right)\right\},
$$

an interval-valued hesitant fuzzy element (IVHFE).

Here $\widetilde{\gamma}=\left[\widetilde{\gamma}^{L}, \widetilde{\gamma}^{U}\right]$ is an interval number. Let $\widetilde{\gamma}^{L}=\inf \widetilde{\gamma}$ and $\widetilde{\gamma}^{U}=\sup \widetilde{\gamma}$ represent the lower and upper limits of $\widetilde{\gamma}$, respectively. An IVHFE is the basic unit of an IVHFS, and it can be considered as a special case of the IVHFS. The relationship between IVHFE and IVHFS is similar to that between interval-valued fuzzy number and interval-valued fuzzy set.

Definition 2. Ref. [28]. Let $\alpha=\left[\alpha^{L}, \alpha^{U}\right]$ and $\beta=\left[\beta^{L}, \beta^{U}\right]$, and let $l_{\alpha}=\alpha^{U}-\alpha^{L}, l_{\beta}=\beta^{U}-\beta^{L}$; then the degree of possibility of $\alpha \geq \beta$ is denoted by

$$
p(\alpha \geq \beta)=\max \left\{1-\max \left(\frac{\beta^{U}-\alpha^{L}}{l_{\alpha}+l_{\beta}}\right), 0\right\},
$$

If $p(\alpha \geq \beta) \geq 0.5$, then called $\alpha$ is greater than $\beta$, that is $\alpha \geq \beta$.

Definition 3. Ref. [28]. For an IVHFE $\widetilde{h}, s(\widetilde{h})=\frac{1}{\# h} \sum_{\gamma \in h} \widetilde{\gamma}$ is called the score function of $\widetilde{h}$ with $\# \widetilde{h}$ being the number of the interval values in $\widetilde{h}$, and $s(\widetilde{h})$ is an interval value belonging to $[0,1]$. For two IVHFEs $\widetilde{h}_{1}$ and $\widetilde{h}_{2}$, if $s\left(\widetilde{h}_{1}\right) \geq s\left(\widetilde{h}_{2}\right)$, then $\widetilde{h}_{1} \geq \widetilde{h}_{2}$. 


\subsection{Probabilistic Interval-Valued Hesitant Fuzzy Sets}

Example 1. Supplier selection is a very important problem in supply chain management. A network dealer invited ten experts to evaluate the comprehensive conditions of a supplier. Among them, four experts' satisfaction interval value of $[0.8,0.9]$, the satisfaction interval value given by two experts is $[0.6,0.8]$, the satisfaction interval value given by three experts is $[0.4,0.6]$, and the remaining experts gave a satisfaction interval value of $[0.3,0.5]$. Using interval-valued hesitant fuzzy element, the above decision information can be expressed as $\{[0.8,0.9],[0.6,0.8],[0.4,0.9],[0.3,0.5]\}$. Obviously, interval-valued hesitant fuzzy elements can only represent all possible decision information, but cannot reflect the importance of each decision information. However, using PIVHFE to represent the above decision information is $\{([0.8,0.9], 0.4)([0.6,0.8], 0.3),([0.4,0.9], 0.2),([0.3,0.5], 0.1)\}$, which comprehensively represents all the evaluation information given by the decision maker. In fact, the given number of real numbers in PIVHFE is not the same, and the element arrangement is unordered in the actual decision problem, so the following rules are made:

Note 1. The intervals in the probabilistic interval-valued hesitant fuzzy element, let PIVHFE $h=\left\{\left(\left[\gamma^{(k)-}, \gamma^{(k)+}\right], p^{(k)}\right) \mid\left[\gamma^{(k)-}, \gamma^{(k)+}\right] \in h\right\}$ be arranged in descending order, which $\left(\left[\gamma^{(k)-}, \gamma^{(k)+}\right], p^{(k)}\right)$ represents the interval corresponding to the membership degree of the largest K.

Note 2. Let $l_{1}$ and $l_{2}$ be the number of elements in probabilistic interval-valued hesitant fuzzy elements (PIVHFE) $h_{1}$ and $h_{2}$ respectively. If $l_{1} \neq l_{2}$, the number of elements can be increased in the PIVHFE with fewer original numbers, so that the number of elements in the two PIVHFE is the same. For the convenience of computation, the number of elements in PIVHFE is assumed to be $l$.

Definition 4. Let $X=\left\{x_{1}, x_{2}, \cdots, x_{m}\right\}$ be a given set. A probabilistic interval-valued hesitant fuzzy sets (PIVHFS) $H$ over $X$ is an object having the form: $H=\left\{\left\langle x_{i}, h\left(x_{i}\right)\right\rangle \mid x_{i} \in X\right\}$, where $h\left(x_{i}\right)=$ $\left\{\left(\left[\gamma_{i}^{-}, \gamma_{i}^{+}\right], p_{\gamma_{i}}\right) \mid\left[\gamma_{i}^{-}, \gamma_{i}^{+}\right] \in h\left(x_{i}\right), p_{\gamma_{i}} \in[0,1], \sum_{\left[\gamma_{i}^{-}, \gamma_{i}^{+}\right] \in h\left(x_{i}\right)} p_{\gamma_{i}}=1\right\}$. here $\gamma_{i}=\left[\gamma_{i}^{-}, \gamma_{i}^{+}\right]$is an interval number. Let $\gamma_{i}^{-}$and $\gamma_{i}^{+}$represent the lower and upper limits of $\gamma_{i}$, respectively. $p_{\gamma_{i}}$ is the probability associated with $\gamma_{i}$.

For convenience, a probabilistic interval-valued hesitant fuzzy element (PIVHFE) will be denoted by $h_{i}=h\left(x_{i}\right)$, and $\bar{P}$ is the set of all PIVHFE on $X$.

The score function for IVHFEs will be defined below.

Definition 5. For a PIVHFE $h$, the score function of $h$ is formulated by

$$
S(h)=\frac{1}{\# h} \sum_{k=1}^{l}\left(\gamma^{(k)}\right) p^{(k)}
$$

with \#h being the number of the interval values in $h$, and $S(h)$ is an interval value belonging to $[0,1]$. For two IVHFEs $h_{1}$ and $h_{2}$, if $S\left(h_{1}\right) \geq S\left(h_{2}\right)$, then $h_{1} \geq h_{2}$. The PIVHFE's deviation function is defined as:

$$
\phi(h)=\sum_{k=1}^{l}\left[0.5\left(\gamma^{(k)-}+\gamma^{(k)+}\right)-S(h)\right]^{2} p^{(k)} .
$$

Definition 6. Let $h_{1}=\left\{\left(\left[\gamma_{1}^{(k)-}, \gamma_{1}^{(k)+}\right], p_{1}^{(k)}\right) \mid k=1,2, \cdots, l\right\}$ and $h_{2}=\left\{\left(\left[\gamma_{2}^{(k)-}, \gamma_{2}^{(k)+}\right], p_{2}^{(k)}\right) \mid k=\right.$ $1,2, \cdots, l\}$ be two PIVHFEs, then:

1. If $S\left(h_{1}\right)>S\left(h_{2}\right)$, then $h_{1}>h_{2}$, that is, $h_{1}$ is greater than $h_{2}$.

2. If $S\left(h_{1}\right)=S\left(h_{2}\right)$, then 
(a) If $\phi\left(h_{1}\right)>\phi\left(h_{2}\right)$, then $h_{1}<h_{2}$. That is, $h_{1}$ is less than $h_{2}$.

(b) If $\phi\left(h_{1}\right)<\phi\left(h_{2}\right)$, then $h_{1}>h_{2}$. That is, $h_{1}$ is greater than $h_{2}$.

(c) If $\phi\left(h_{1}\right)=\phi\left(h_{2}\right)$, then $h_{1}=h_{2}$. That is, $h_{1}$ equals $h_{2}$.

\subsection{Archimedean Norm}

Most of the existing information on aggregation operators are based on algebraic multiplication and addition operations, but the algebraic multiplication and addition operations only in a form of Archimedean norm [30,31].

Related studies show that, the strict Archimedean T-norm can be obtained by a strictly monotone decreasing additive operator $g:[0,1] \rightarrow[0,+\infty]$ is expressed as $T(x, y)=g^{-1}(g(x)+g(y))$, where $g(1)=0, g(0)=1$. According to duality principle, the strict Archimedean S-norm can be expressed as $S(x, y)=f^{-1}(f(x)+f(y))$, where $f(t)=g(1-t)$, Thus, $f(t)$ strictly monotonically increases, and $f(0)=0, f(1)=1$.

\subsection{Probabilistic Interval-Valued Hesitant Fuzzy Operation Rules}

In the probabilistic interval-valued hesitant fuzzy information environment, the following operation laws are introduced based on the Archimedean norm.

Definition 7. Let $h, h_{1}$ and $h_{2}$ be three PIVHFEs, then (For convenience, let $k=1,2, \cdots, l, \lambda>0$ be omitted in the following sets):

(1) $h^{c}=\left\{\left(\left[1-\gamma^{(k)-}, 1-\gamma^{(k)+}\right], p^{(k)}\right)\right\}$

(2) $h_{1} \oplus h_{2}=\left\{\left(S\left(\left[\gamma_{1}^{(k)-}, \gamma_{1}^{(k)+}\right],\left[\gamma_{2}^{(k)-}, \gamma_{2}^{(k)+}\right]\right), \overline{p_{1}^{(k)}+p_{2}^{(k)}}\right)\right\}=\left\{\left(\left[f^{-1}\left(f\left(\gamma_{1}^{(k)-}\right)+\right.\right.\right.\right.$ $\left.\left.\left.\left.f\left(\gamma_{2}^{(k)-}\right)\right), f^{-1}\left(f\left(\gamma_{1}^{(k)+}\right)+f\left(\gamma_{2}^{(k)+}\right)\right)\right], \overline{p_{1}^{(k)}+p_{2}^{(k)}}\right)\right\}$

(3) $h_{1} \otimes h_{2}=\left\{\left(T\left(\left[\gamma_{1}^{(k)-}, \gamma_{1}^{(k)+}\right],\left[\gamma_{2}^{(k)-}, \gamma_{2}^{(k)+}\right]\right), \overline{p_{1}^{(k)}+p_{2}^{(k)}}\right)\right\}=\left\{\left(\left[g^{-1}\left(g\left(\gamma_{1}^{(k)-}\right)+\right.\right.\right.\right.$ $\left.\left.\left.\left.g\left(\gamma_{2}^{(k)-}\right)\right), g^{-1}\left(g\left(\gamma_{1}^{(k)+}\right)+g\left(\gamma_{2}^{(k)+}\right)\right)\right], \overline{p_{1}^{(k)}+p_{2}^{(k)}}\right)\right\}$

(4) $\lambda h=\left\{\left(\left[f^{-1}\left(\lambda f\left(\gamma^{(k)-}\right)\right), f^{-1}\left(\lambda f\left(\gamma^{(k)+}\right)\right)\right], p^{(k)}\right)\right\}$

(5) $\quad h^{\lambda}=\left\{\left(\left[g^{-1}\left(\lambda g\left(\gamma^{(k)-}\right)\right), g^{-1}\left(\lambda g\left(\gamma^{(k)+}\right)\right)\right], p^{(k)}\right)\right\}$

where $\overline{p_{1}^{(k)}+p_{2}^{(k)}}=\left(p_{1}^{(k)}+p_{2}^{(k)}\right) /\left(\sum_{k=1}^{l}\left(p_{1}^{(k)}+p_{2}^{(k)}\right)\right), k=1,2, \cdots, l$, then $\sum_{k=1}^{l}\left(\overline{p_{1}^{(k)}+p_{2}^{(k)}}\right)=1$.

It is easy to prove that the above probabilistic interval-valued hesitant fuzzy operation rules proposed satisfy the following properties.

Theorem 1. Let $h, h_{1}$ and $h_{2}$ be three PIVHFEs, then:

(1) $h_{1} \oplus h_{2}=h_{2} \oplus h_{1}$;

(2) $h_{1} \otimes h_{2}=h_{2} \otimes h_{1}$;

(3) $\lambda\left(h_{1} \oplus h_{2}\right)=\lambda h_{1} \oplus \lambda h_{2}, \lambda>0$;

(4) $\left(h_{1} \otimes h_{2}\right)^{\lambda}=h_{1}^{\lambda} \otimes h_{2}^{\lambda}, \lambda>0$;

(5) $\lambda_{1} h \oplus \lambda_{2} h=\left(\lambda_{1}+\lambda_{2}\right) h, \lambda_{1}, \lambda_{2}>0$;

(6) $h^{\lambda_{1}} \otimes h^{\lambda_{2}}=h^{\lambda_{1}+\lambda_{2}}, \lambda_{1}, \lambda_{2}>0$.

\section{Generalized Probabilistic Interval-Valued Hesitant Fuzzy Information Aggregation Operators}

In the actual MAGDM problem, in order to comprehensively and accurately reflect the attribute information and the importance degree of decision makers, PIVHFE will be used to represent the decision information under the attribute of the program. Then, the information aggregation operators will be used to fuse all the attribute values under a certain scheme to obtain the comprehensive attribute information. Therefore, based on the new algorithms in Definition 6, the generalized probabilistic 
interval-valued hesitant fuzzy ordered weighted averaging (GPIVHFOWA) operator and the generalized probabilistic interval-valued hesitant fuzzy ordered weighted geometric (GPIVHFOWG) operator are proposed in this section, and then the related properties are investigated.

\subsection{GPIVHFOWA Operator}

Definition 8. Let $h_{j}(j=1,2, \cdots, n)$ be a set of PIVHFEs, a generalized probabilistic interval-valued hesitant fuzzy ordered weighted averaging (GPIVHFOWA) operator is a mapping GPIVHFOWA: $H^{n} \rightarrow H$, such that

$$
\operatorname{GPIVHFOWA}\left(h_{1}, h_{2}, \cdots, h_{n}\right)=\bigoplus_{j=1}^{n} w_{j} h_{\sigma(j)},
$$

where let $w=\left(w_{1}, w_{2}, \cdots, w_{n}\right)^{T}$ be the related weight vectors, and satisfies the condition $w_{j} \geq 0$ and $\sum_{j=1}^{n} w_{j}=1$.

Theorem 2. Let $h_{j}(j=1,2, \cdots, n)$ be a set of PIVHFEs, then the aggregated value by the GPIVHFOWA operator is also PIVHFE, and

$$
\operatorname{GPIVHFOWA}\left(h_{1}, h_{2}, \cdots, h_{n}\right)={\underset{j=1}{\oplus} w_{j} h_{\sigma(j)}}^{n}\left\{\left(\left[f^{-1}\left(\sum_{j=1}^{n} w_{j} f\left(\gamma_{\sigma(j)}^{(k)-}\right)\right), f^{-1}\left(\sum_{j=1}^{n} w_{j} f\left(\gamma_{\sigma(j)}^{(k)+}\right)\right)\right], \overline{\sum_{j=1}^{n} p_{j}^{(k)}}\right) \mid k=1,2, \cdots, l\right\},
$$

where $\left[\gamma_{\sigma(j)}^{(k)-}, \gamma_{\sigma(j)}^{(k)+}\right]$ is the $j$ interval in descending order of $\left[\gamma_{1}^{(k)-}, \gamma_{1}^{(k)+}\right],\left[\gamma_{2}^{(k)-}, \gamma_{2}^{(k)+}\right], \cdots,\left[\gamma_{n}^{(k)-}, \gamma_{n}^{(k)+}\right]$, and $\overline{\sum_{j=1}^{n} p_{j}^{(k)}}=\sum_{j=1}^{n} p_{j}^{(k)} / \sum_{k=1}^{l} \sum_{j=1}^{n} p_{j}^{(k)}$.

\section{Proof.}

(1) It is proved that the aggregation result obtained by an operator is still PIVHFE, then

$$
\left[f^{-1}\left(\sum_{j=1}^{n} w_{j} f\left(\gamma_{\sigma(j)}^{(k)-}\right)\right), f^{-1}\left(\sum_{j=1}^{n} w_{j} f\left(\gamma_{\sigma(j)}^{(k)+}\right)\right)\right] \in[0,1], \overline{\sum_{j=1}^{n} p_{j}^{(k)}} \in[0,1], \sum_{k=1}^{l} \overline{\sum_{j=1}^{n} p_{j}^{(k)}}=1 .
$$

According to the computational formula of $\overline{\sum_{j=1}^{n} p_{j}^{(k)}}$, we know that $\overline{\sum_{j=1}^{n} p_{j}^{(k)}} \in[0,1], \sum_{k=1}^{l} \overline{\sum_{j=1}^{n} p_{j}^{(k)}}=1$ are clearly established.

Since $0 \leq \gamma_{\sigma(j)}^{(k)-} \leq \gamma_{\sigma(j)}^{(k)+} \leq 1$ for all $k=1,2, \cdots, l, j=1,2, \cdots, n . f(t)$ and $f^{-1}(t)$ are strictly monotone increasing functions, then we have $f(0) \leq f\left(\gamma_{\sigma(j)}^{(k)-}\right) \leq f\left(\gamma_{\sigma(j)}^{(k)+}\right) \leq f(1)$, and then $w_{j} f(0) \leq$ $w_{j} f\left(\gamma_{\sigma(j)}^{(k)-}\right) \leq w_{j} f\left(\gamma_{\sigma(j)}^{(k)+}\right) \leq w_{j} f(1)$.

It pointed out that

$$
f(0)=\sum_{j=1}^{n} w_{j} f(0) \leq \sum_{j=1}^{n} w_{j} f\left(\gamma_{\sigma(j)}^{(k)-}\right) \leq \sum_{j=1}^{n} w_{j} f\left(\gamma_{\sigma(j)}^{(k)+}\right) \leq \sum_{j=1}^{n} w_{j} f(1)=f(1) .
$$

The simultaneous acting function $f^{-1}(t)$ on both sides of the above formula, then

$$
\begin{gathered}
0=f^{-1}(f(0)) \leq f^{-1}\left(\sum_{j=1}^{n} w_{j} f\left(\gamma_{\sigma(j)}^{(k)-}\right)\right) \leq f^{-1}\left(\sum_{j=1}^{n} w_{j} f\left(\gamma_{\sigma(j)}^{(k)+}\right)\right) \leq f^{-1}(f(1))=1, \\
{\left[f^{-1}\left(\sum_{j=1}^{n} w_{j} f\left(\gamma_{\sigma(j)}^{(k)-}\right)\right), f^{-1}\left(\sum_{j=1}^{n} w_{j} f\left(\gamma_{\sigma(j)}^{(k)+}\right)\right)\right] \in[0,1] .}
\end{gathered}
$$

Therefore, our proof of Theorem 2 is over. 
(2) Next, the mathematical induction will be used to prove the Equation (7). When $n=2$, we have:

$$
\begin{aligned}
& \text { GPIVHFOWA }\left(h_{1}, h_{2}\right)=w_{1} h_{\sigma(1)} \oplus w_{2} h_{\sigma(2)} \\
& =\left\{\left(\left[f^{-1}\left(w_{1} f\left(\gamma_{\sigma(1)}^{(k)-}\right)\right), f^{-1}\left(w_{1} f\left(\gamma_{\sigma(1)}^{(k)}\right)\right)\right], p_{1}^{(k)}\right) \mid k=1,2, \cdots, l\right\} \oplus\left\{\left(\left[f^{-1}\left(w_{2} f\left(\gamma_{\sigma(2)}^{(k)}\right)\right), f^{-1}\left(w_{2} f\left(\gamma_{\sigma(2)+}^{(k)}\right)\right)\right], p_{2}^{(k)}\right) \mid k=1,2, \cdots, l\right\} \\
& =\left\{\left(\left[f^{-1}\left(f\left(f^{-1}\left(w_{1} f\left(\gamma_{\sigma(1)}^{(k)-}\right)\right)\right)+f\left(f^{-1}\left(w_{2} f\left(\gamma_{\sigma(2)}^{(k)}\right)\right)\right)\right), f^{-1}\left(f\left(f^{-1}\left(w_{1} f\left(\gamma_{\sigma(1)}^{(k)+}\right)\right)\right)+f\left(f^{-1}\left(w_{2} f\left(\gamma_{\sigma(2)}^{(k)+}\right)\right)\right)\right], p_{1}^{(k)}+p_{2}^{(k)}\right) \mid k=1,2, \cdots, l\right\}\right. \\
& =\left\{\left(\left[f^{-1}\left(w_{1} f\left(\gamma_{\sigma(1)}^{(k)-}\right)+w_{2} f\left(\gamma_{\sigma(2)}^{(k)-}\right)\right), f^{-1}\left(w_{1} f\left(\gamma_{\sigma(1)}^{(k)+}\right)+w_{2} f\left(\gamma_{\sigma(2)}^{(k)+}\right)\right)\right], \overline{\left.p_{1}^{(k)}+p_{2}^{(k)}\right)}\right) \mid k=1,2, \cdots, l\right\}
\end{aligned}
$$

So, when $n=2$, Equation (7) is established. Suppose Equation (7) holds for $\mathrm{n}=\mathrm{q}$, that is:

$$
\left.\operatorname{GPIVHFOWA}\left(h_{1}, h_{2}, \cdots, h_{q}\right)=\underset{j=1}{q} w_{j} h_{\sigma(j)}=\left\{\left[f^{-1}\left(\sum_{j=1}^{q} w_{j} f\left(\gamma_{\sigma(j)}^{(k)}\right)\right), f^{-1}\left(\sum_{j=1}^{q} w_{j} f\left(\gamma_{\sigma(j)}^{(k)+}\right)\right)\right], \overline{\sum_{j=1}^{q} p_{j}^{(k)}}\right) \mid k=1,2, \cdots, l\right\},
$$

Then, when $n=q+1$, we have

$$
\begin{aligned}
& \operatorname{GPIVHFOWA}\left(h_{1}, h_{2}, \cdots, h_{q+1}\right)=\underset{\Theta=1}{q+1} w_{j} h_{\sigma(j)}=\left(\underset{\substack{\oplus \\
j=1}}{q} w_{j} h_{\sigma(j)}\right) \oplus w_{q+1} h_{\sigma(q+1)} \\
& =\left\{\left(\left[f^{-1}\left(\sum_{j=1}^{q} w_{j} f\left(\gamma_{\sigma(j)}^{(k)}\right)\right), f^{-1}\left(\sum_{j=1}^{q} w_{j} f\left(\gamma_{\sigma(j)}^{(k)+}\right)\right)\right], \sum_{j=1}^{q} p_{j}^{(k)}\right) \mid k=1,2, \cdots, l\right\} \oplus\left\{\left(\left[f^{-1}\left(w_{q+1} f\left(\gamma_{\sigma(q+1)}^{(k)}\right)\right), f^{-1}\left(w_{q+1} f\left(\gamma_{\sigma(q+1)}^{(k)+}\right)\right)\right], p_{q+1}^{(k)}\right) \mid k=1,2, \cdots, l\right\} \\
& =\left\{\left(\left[f^{-1}\left(f\left(f^{-1}\left(\sum_{j=1}^{q} w_{j} f\left(\gamma_{\sigma(j)}^{(k)}\right)\right)\right)+f\left(f^{-1}\left(w_{q+1} f\left(\gamma_{\sigma(q)+1}^{(k)}\right)\right)\right), f^{-1}\left(f\left(f^{-1}\left(\sum_{j=1}^{q} w_{j} f\left(\gamma_{\sigma(j)}^{(k)+}\right)\right)\right)+f\left(f^{-1}\left(w_{q+1} f\left(\gamma_{\sigma(q+1)}^{(k)+}\right)\right)\right)\right)\right], \sum_{j=1}^{\overline{q+1}} p_{j}^{(k)}\right) \mid k=1,2, \cdots, l\right\}\right. \\
& \left.=\left\{\left(\left[f^{-1}\left(\sum_{j=1}^{q} w_{j} f\left(\gamma_{\sigma())}^{(k)-}\right)+w_{q+1} f\left(\gamma_{\sigma(q+1)}^{(k)-}\right)\right), f^{-1}\left(\sum_{j=1}^{q} w_{j} f\left(\gamma_{\sigma(j)}^{(k)+}\right)+w_{q+1} f\left(\gamma_{\sigma(q+1)}^{(k)+}\right)\right)\right],\right]_{j=1}^{q+1} p_{j}^{(k)}\right) \mid k=1,2, \cdots, l\right\} \\
& =\left\{\left(\left[f^{-1}\left(\sum_{j=1}^{q+1} w_{j} f\left(\gamma_{\sigma(j)}^{(k)}\right)\right), f^{-1}\left(\sum_{j=1}^{q+1} w_{j} f\left(\gamma_{\sigma(j)}^{(k)+}\right)\right)\right], \sum_{j=1}^{q+1} p_{j}^{(k)}\right) \mid k=1,2, \cdots, l\right\}
\end{aligned}
$$

Therefore Equation (7) holds for all $n$, and our proof of Theorem 3 is over.

\subsection{The GPIVHFOWG Operator}

Definition 9. Let $h_{j}(j=1,2, \cdots, n)$ be a set of PIVHFEs, a generalized probabilistic interval-valued hesitant fuzzy ordered weighted geometric (GPIVHFOWG) operator is a mapping GPIVHFOWG: $H^{n} \rightarrow H$, such that

$$
\operatorname{GPIVHFOWG}\left(h_{1}, h_{2}, \cdots, h_{n}\right)=\bigotimes_{j=1}^{n} w_{j} h_{\sigma(j)},
$$

where let $w=\left(w_{1}, w_{2}, \cdots, w_{n}\right)^{\mathrm{T}}$ be the related weight vectors, and satisfies the condition $w_{j} \geq 0$ and $\sum_{j=1}^{n} w_{j}=1$.

Theorem 3. Let $h_{j}(j=1,2, \cdots, n)$ be a set of PIVHFEs, then the aggregated value by the GPIVHFOWG operator is also an PIVHFE, and

$$
\left.\operatorname{GPIVHFOWG}\left(h_{1}, h_{2}, \cdots, h_{n}\right)={\underset{j=1}{\otimes}}_{j=1}^{n} w_{j} h_{\sigma(j)}=\left\{\left[g^{-1}\left(\sum_{j=1}^{n} w_{j} g\left(\gamma_{\sigma(j)}^{(k)-}\right)\right), g^{-1}\left(\sum_{j=1}^{n} w_{j} g\left(\gamma_{\sigma(j)}^{(k)+}\right)\right)\right], \overline{\sum_{j=1}^{n} p_{j}^{(k)}}\right) \mid k=1,2, \cdots, l\right\},
$$

where $\left[\gamma_{\sigma(j)}^{(k)-}, \gamma_{\sigma(j)}^{(k)+}\right]$ is the jinterval in descending order of $\left[\gamma_{1}^{(k)-}, \gamma_{1}^{(k)+}\right],\left[\gamma_{2}^{(k)-}, \gamma_{2}^{(k)+}\right], \cdots,\left[\gamma_{n}^{(k)-}, \gamma_{n}^{(k)+}\right]$, and $\overline{\sum_{j=1}^{n} p_{j}^{(k)}}=\sum_{j=1}^{n} p_{j}^{(k)} / \sum_{k=1}^{l} \sum_{j=1}^{n} p_{j}^{(k)}$.

The proof of Theorem 3 is similar to Theorem 2, therefore it is omitted.

\subsection{The Properties of the GPIVHFOWA Operator and the GPIVHFOWG Operator}

This section will explore some basic properties of the GPIVHFOWA operator and the GPIVHFOWG operator, including idempotency, monotonicity and boundedness and commutativity.

Property 1 (idempotency). Let $h_{j}(j=1,2, \cdots, n)$ be a set of PIVHFEs. If $\forall i=1,2, \cdots, n, h_{i}=h$, then:

$$
\text { GPIVHFOWA }\left(h_{1}, h_{2}, \cdots, h_{n}\right)=h \text {, }
$$




$$
\text { GPIVHFOWG }\left(h_{1}, h_{2}, \cdots, h_{n}\right)=h,
$$

where $w=\left(w_{1}, w_{2}, \cdots, w_{n}\right)^{\mathrm{T}}$ is the weighted vector of $h_{j}$, with $w_{j} \geq 0$ and $\sum_{j=1}^{n} w_{j}=1$.

Proof. When $h_{1}=h_{2}=\cdots=h_{n}=h=\left\{\left(\left[\gamma^{(k)-}, \gamma^{(k)+}\right], p^{(k)}\right) \mid k=1,2, \cdots, l\right\}$, since $\sum_{k=1}^{l} p^{(k)}=1, l=1,2, \cdots, n$, then $\left[f^{-1}\left(\sum_{j=1}^{n} w_{j} f\left(\gamma_{\sigma(j)}^{(k)-}\right)\right), f^{-1}\left(\sum_{j=1}^{n} w_{j} f\left(\gamma_{\sigma(j)}^{(k)+}\right)\right)\right]=$ $\left[f^{-1}\left(\sum_{j=1}^{n} w_{j} f\left(\gamma^{(k)-}\right)\right), f^{-1}\left(\sum_{j=1}^{n} w_{j} f\left(\gamma^{(k)+}\right)\right)\right]$
$\quad=\left[f^{-1}\left(f\left(\gamma^{(k)-}\right) \sum_{j=1}^{n} w_{j}\right), f^{-1}\left(f\left(\gamma^{(k)+}\right) \sum_{j=1}^{n} w_{j}\right)\right]=\left[f^{-1}\left(f\left(\gamma^{(k)-}\right)\right), f^{-1}\left(f\left(\gamma^{(k)+}\right)\right)\right]=\left[\gamma^{(k)-}, \gamma^{(k)+}\right]$,

and

$$
\begin{aligned}
& {\left[g^{-1}\left(\sum_{j=1}^{n} w_{j} g\left(\gamma_{\sigma(j)}^{(k)-}\right)\right), g^{-1}\left(\sum_{j=1}^{n} w_{j} g\left(\gamma_{\sigma(j)}^{(k)+}\right)\right)\right]=\left[g^{-1}\left(\sum_{j=1}^{n} w_{j} g\left(\gamma^{(k)-}\right)\right), g^{-1}\left(\sum_{j=1}^{n} w_{j} g\left(\gamma^{(k)+}\right)\right)\right] } \\
&= {\left[g^{-1}\left(g\left(\gamma^{(k)-}\right) \sum_{j=1}^{n} w_{j}\right), g^{-1}\left(g\left(\gamma^{(k)+}\right) \sum_{j=1}^{n} w_{j}\right)\right]=\left[g^{-1}\left(g\left(\gamma^{(k)-}\right)\right), g^{-1}\left(g\left(\gamma^{(k)+}\right)\right)\right]=\left[\gamma^{(k)-}, \gamma^{(k)+}\right], } \\
& \overline{\sum_{j=1}^{n} p_{j}^{(k)}}=\sum_{j=1}^{n} p_{j}^{(k)} / \sum_{k=1}^{l} \sum_{j=1}^{n} p_{j}^{(k)}=\sum_{j=1}^{n} p^{(k)} / \sum_{j=1}^{n} \sum_{k=1}^{l} p^{(k)}=n p^{(k)} / \sum_{j=1}^{n} 1=\frac{n p^{(k)}}{n}=p^{(k)} .
\end{aligned}
$$

In summary, our proof of Property 1 is over.

Property 2 (monotonicity). Let $h_{j}=\left\{\left(\left[\gamma_{j}^{(k)-}, \gamma_{j}^{(k)+}\right], p_{j}^{(k)}\right) \mid k=1,2, \cdots, l\right\}$ and $a_{j}=$ $\left\{\left(\left[\eta_{j}^{(k)-}, \eta_{j}^{(k)+}\right], q_{j}^{(k)}\right) \mid k=1,2, \cdots, l\right\}(j=1,2, \cdots, n)$ be two sets of PIVHFEs. If $\forall k=1,2, \cdots, l$, $j=1,2, \cdots, n$, then $\gamma_{i}^{(k)-} \leq \gamma_{i}^{(k)+} \leq \eta_{i}^{(k)-} \leq \eta_{i}^{(k)+}, p_{i}^{(k)} \leq q_{i}^{(k)}$. It follows that

$$
\begin{aligned}
& \operatorname{GPIVHFOWA}\left(h_{1}, h_{2}, \cdots, h_{n}\right) \leq \operatorname{GPIVHFOWA}\left(a_{1}, a_{2}, \cdots, a_{n}\right), \\
& \operatorname{GPIVHFOWG}\left(h_{1}, h_{2}, \cdots, h_{n}\right) \leq \operatorname{GPIVHFOWG}\left(a_{1}, a_{2}, \cdots, a_{n}\right),
\end{aligned}
$$

where $w=\left(w_{1}, w_{2}, \cdots, w_{n}\right)^{T}$ is associated weighted vector such that $w_{j} \geq 0, \sum_{j=1}^{n} w_{j}=1$.

Thus, the combination of Property 1 and Property 2, the following properties will be proved.

Property 3 (boundedness). Let $h_{j}=\left\{\left[\gamma_{j}^{(k)-}, \gamma_{j}^{(k)+}\right], p_{j}^{(k)} \mid k=1,2, \cdots, l\right\}$ be a set of PIVHFEs. If $h^{-}=$ $\left\{\left(\left[\min _{j} \gamma_{j}^{(k)-}, \min _{j} \gamma_{j}^{(k)+}\right], \min _{j} p_{j}^{(k)}\right) \mid k=1,2, \cdots, l\right\} h^{+}=\left\{\left(\left[\max _{j} \gamma_{j}^{(k)-}, \max _{j} \gamma_{j}^{(k)+}\right], \max _{j} p_{j}^{(k)}\right) \mid k=\right.$ $1,2, \cdots, l\}$, then

$$
\begin{aligned}
& h^{-} \leq \operatorname{GPIVHFOWA}\left(h_{1}, h_{2}, \cdots, h_{n}\right) \leq h^{+}, \\
& h^{-} \leq \operatorname{GPIVHFOWG}\left(h_{1}, h_{2}, \cdots, h_{n}\right) \leq h^{+},
\end{aligned}
$$

where $w=\left(w_{1}, w_{2}, \cdots, w_{n}\right)^{\mathrm{T}}$ is the weighted vector of $h_{j}$, with $w_{j} \geq 0$ and $\sum_{j=1}^{n} w_{j}=1$.

Property 4 (commutativity). Let $h_{j}(j=1,2, \cdots, n)$ be a set of PIVHFEs and $w=\left(w_{1}, w_{2}, \cdots, w_{n}\right)^{\mathrm{T}}$ be the weighted vector of $h_{j}$, with $w_{j} \geq 0$ and $\sum_{j=1}^{n} w_{j}=1$, then

$$
\begin{aligned}
& \operatorname{GPIVHFOWA}\left(h_{1}, h_{2}, \cdots, h_{n}\right)=\operatorname{GPIVHFOWA}\left(\widetilde{h}_{1}, \widetilde{h}_{2}, \cdots, \widetilde{h}_{n}\right), \\
& \operatorname{GPIVHFOWG}\left(h_{1}, h_{2}, \cdots, h_{n}\right)=\operatorname{GPIVHFOWG}\left(\widetilde{h}_{1}, \widetilde{h}_{2}, \cdots, \widetilde{h}_{n}\right),
\end{aligned}
$$


where $\left(\widetilde{h}_{1}, \widetilde{h}_{2}, \cdots, \widetilde{h}_{n}\right)$ is any permutation of $\left(h_{1}, h_{2}, \cdots, h_{n}\right)$.

\section{Relationship among the Probabilistic Interval-Valued Hesitant Fuzzy Information Aggregation Operators}

In this section, we first study some common information aggregation operators when additive operator $g(t)$ take different functions. Then, the relationships among these common information aggregation operators are discussed.

Case 1: When $g(t)=-\ln (t)$, the GPIVHFOWA operator and the GPIVHFOWG operator are transformed into probabilistic interval-valued hesitant fuzzy ordered weighted averaging (PIVHFOWA) operators and probabilistic interval-valued hesitant fuzzy ordered weighted geometric (PIVHFOWG) operators respectively, as follows:

$$
\begin{aligned}
& \operatorname{PIVHFOWA}\left(h_{1}, h_{2}, \cdots, h_{n}\right)=\left\{\left(\left[1-\prod_{j=1}^{n}\left(1-\gamma_{\sigma(j)}^{(k)-}\right)^{w_{j}}, 1-\prod_{j=1}^{n}\left(1-\gamma_{\sigma(j)}^{(k)+}\right)^{w_{j}}\right], \overline{\sum_{j=1}^{n} p_{j}^{(k)}}\right) \mid k=1,2, \cdots, l\right\}, \\
& \operatorname{PIVHFOWG}\left(h_{1}, h_{2}, \cdots, h_{n}\right)=\left\{\left(\left[\prod_{j=1}^{n}\left(\gamma_{\sigma(j)}^{(k)-}\right)^{w_{j}}, \prod_{j=1}^{n}\left(\gamma_{\sigma(j)}^{(k)+}\right)^{w_{j}}\right], \overline{\sum_{j=1}^{n} p_{j}^{(k)}}\right) \mid k=1,2, \cdots, l\right\},
\end{aligned}
$$

Similar operators are proposed in the interval-valued hesitant fuzzy environment of literature [28], namely interval-valued hesitant fuzzy ordered weighted averaging (IVHFOWA) operator and interval-valued hesitant fuzzy ordered weighted geometric (IVHFOWG) operator, as follows:

$$
\begin{aligned}
& \operatorname{IVHFOWA}\left(\widetilde{h}_{1}, \widetilde{h}_{2}, \cdots, \widetilde{h}_{n}\right)=\bigoplus_{j=1}^{n}\left(w_{j} \widetilde{h}_{\sigma(j)}\right) \\
& =\left\{\left[1-\prod_{j=1}^{n}\left(1-\widetilde{\gamma}_{\sigma(j)}^{L}\right)^{w_{j}}, 1-\prod_{j=1}^{n}\left(1-\widetilde{\gamma}_{\sigma(j)}^{U}\right)^{w_{j}}\right] \mid \widetilde{\gamma}_{\sigma(1)} \in \widetilde{h}_{\sigma(1)}, \widetilde{\gamma}_{\sigma(2)} \in \widetilde{h}_{\sigma(2)}, \cdots, \widetilde{\gamma}_{\sigma(n)} \in \widetilde{h}_{\sigma(n)}\right\}, \\
& \operatorname{IVHFOWG}\left(\widetilde{h}_{1}, \widetilde{h}_{2}, \cdots, \widetilde{h}_{n}\right)=\bigotimes_{j=1}^{n}\left(w_{j} \widetilde{h}_{\sigma(j)}\right) \\
& =\left\{\left[\prod_{j=1}^{n}\left(\widetilde{\gamma}_{\sigma(j)}^{L}\right)^{w_{j}}, \prod_{j=1}^{n}\left(\widetilde{\gamma}_{\sigma(j)}^{U}\right)^{w_{j}}\right] \mid \widetilde{\gamma}_{\sigma(1)} \in \widetilde{h}_{\sigma(1)}, \widetilde{\gamma}_{\sigma(2)} \in \widetilde{h}_{\sigma(2)}, \cdots, \widetilde{\gamma}_{\sigma(n)} \in \widetilde{h}_{\sigma(n)}\right\},
\end{aligned}
$$

Case 2: When $g(t)=-\ln [(2-t) / t]$, the GPIVHFOWA operator and GPIVHFOWG operator are transformed into probabilistic interval-valued hesitant fuzzy Einstein ordered weighted averaging (PIVHFEOWA) operator and the probabilistic interval-valued hesitant fuzzy Einstein ordered weighted geometric (PIVHFEOWG) operator, as follows:

$$
\begin{aligned}
& \text { PIVHFEOWA }\left(h_{1}, h_{2}, \cdots, h_{n}\right) \\
& =\left\{\left(\left[\frac{\prod_{j=1}^{n}\left(1+\gamma_{\sigma(j)}^{(k)}\right)_{j}^{w_{j}}-\prod_{j=1}^{n}\left(1-\gamma_{\sigma(j)}^{(k)}\right)^{w_{j}}}{\prod_{j=1}^{n}\left(1+\gamma_{\sigma(j)}^{(k)}\right)^{w_{j}}+\prod_{j=1}^{n}\left(1-\gamma_{\sigma(j)}^{(k)}\right)^{w_{j}}}, \frac{\prod_{j=1}^{n}\left(1+\gamma_{\sigma(j)}^{(k)+}\right)^{w_{j}}-\prod_{j=1}^{n}\left(1-\gamma_{\sigma(j)}^{(k)+}\right)^{w_{j}}}{\prod_{j=1}^{n}\left(1+\gamma_{\sigma(j)}^{(k)+}\right)^{w_{j}}+\prod_{j=1}^{n}\left(1-\gamma_{\sigma(j)}^{(k)+}\right)^{w_{j}}}\right], \overline{\sum_{j=1}^{n} p_{j}^{(k)}}\right) \mid k=1,2, \cdots, l\right\},
\end{aligned}
$$

$$
\begin{aligned}
& \text { PIVHFEOWG }\left(h_{1}, h_{2}, \cdots, h_{n}\right) \\
& =\left\{\left(\left[\frac{2 \prod_{j=1}^{n}\left(\gamma_{\sigma(j)}^{(k)-}\right)^{w_{j}}}{\prod_{j=1}^{n}\left(2-\gamma_{\sigma(j)}^{(k)-}\right)^{w_{j}}+\prod_{j=1}^{n}\left(\gamma_{\sigma(j)}^{(k)-}\right)^{w_{j}}}, \frac{2 \prod_{j=1}^{n}\left(\gamma_{\sigma(j)}^{(k)+}\right)^{w_{j}}}{\prod_{j=1}^{n}\left(2-\gamma_{\sigma(j)}^{(k)+}\right)^{w_{j}}+\prod_{j=1}^{n}\left(\gamma_{\sigma(j)}^{(k)+}\right)^{w_{j}}}, \overline{\sum_{j=1}^{n} p_{j}^{(k)}}\right) \mid k=1,2, \cdots, l\right\},\right.
\end{aligned}
$$

To analyze the relationship among the PIVHFOWA operator, PIVHFOWG operator, PIVHFEOWA operator and PIVHFEOWG operator, we introduce the following lemma:

Lemma 1. Ref. [32]. Let $b_{j} \geq 0, \mu_{j} \geq 0, j=1,2, \cdots, n$ and $\sum_{j=1}^{n} \mu_{j}=1$, then $\prod_{j=1}^{n} b_{j}^{\mu_{j}} \leq \sum_{j=1}^{n} \mu_{j} b_{j}$.

If $b_{1}=b_{2}=\cdots=b_{n}$, the equality is established.

Based on Lemma 1, we have the following result: 
Theorem 4. Let $h_{j}(j=1,2, \cdots, n)$ be a set of PIVHFEs and $w=\left(w_{1}, w_{2}, \cdots, w_{n}\right)^{\mathrm{T}}$ be the weighted vector of $h_{j}$, with $w_{j} \geq 0$ and $\sum_{j=1}^{n} w_{j}=1$. As follows:

$$
\operatorname{PIVHFOWG}\left(h_{1}, h_{2}, \cdots, h_{n}\right) \leq \operatorname{PIVHFEOWG}\left(h_{1}, h_{2}, \cdots, h_{n}\right),
$$

If $h_{1}=h_{2}=\cdots=h_{n}$, the equality is established.

Proof. For any $k=1,2, \cdots, l$, combining the Lemma 1 , we have

$$
\begin{gathered}
\prod_{j=1}^{n}\left(2-\gamma_{\sigma(j)}^{(k)-}\right)^{w_{j}}+\prod_{j=1}^{n}\left(\gamma_{\sigma(j)}^{(k)-}\right)^{w_{j}} \leq \sum_{j=1}^{n} w_{j}\left(2-\gamma_{\sigma(j)}^{(k)-}\right)^{w_{j}}+\sum_{j=1}^{n} w_{j}\left(\gamma_{\sigma(j)}^{(k)-}\right)^{w_{j}}=2, \text { then } \\
\frac{2 \prod_{j(1}^{n}\left(\gamma_{\sigma(j)}^{(k)-}\right)^{w_{j}}}{\prod_{j=1}^{n}\left(2-\gamma_{\sigma(j)}^{(k)-}\right)^{w_{j}}+\prod_{j=1}^{n}\left(\gamma_{\sigma(j)}^{(k)-}\right)^{w_{j}}} \geq \frac{2 \prod_{j=1}^{n}\left(\gamma_{\sigma(j)}^{(k)-}\right)^{w_{j}}}{2}=\prod_{j=1}^{n}\left(\gamma_{\sigma(j)}^{(k)-}\right)^{w_{j}} .
\end{gathered}
$$

If $\gamma_{\sigma(j)}^{(1)-}=\gamma_{\sigma(j)}^{(2)-}=\cdots=\gamma_{\sigma(j)}^{(l)-}$, the equality is established. Similarly, we have

$$
\frac{2 \prod_{j=1}^{n}\left(\gamma_{\sigma(j)}^{(k)+}\right)^{w_{j}}}{\prod_{j=1}^{n}\left(2-\gamma_{\sigma(j)}^{(k)+}\right)^{w_{j}}+\prod_{j=1}^{n}\left(\gamma_{\sigma(j)}^{(k)+}\right)^{w_{j}}} \geq \frac{2 \prod_{j=1}^{n}\left(\gamma_{\sigma(j)}^{(k)+}\right)^{w_{j}}}{2}=\prod_{j=1}^{n}\left(\gamma_{\sigma(j)}^{(k)+}\right)^{w_{j}} .
$$

If $\gamma_{\sigma(j)}^{(1)+}=\gamma_{\sigma(j)}^{(2)+}=\cdots=\gamma_{\sigma(j)}^{(l)+}$, the equality is established.

Then by Definition 2 and Definition 3, we know that the score function of $\operatorname{PIVHFOWG}\left(h_{1}, h_{2}, \cdots, h_{n}\right)$ is less than the score function of PIVHFEOWG $\left(h_{1}, h_{2}, \cdots, h_{n}\right)$, i.e.,

$$
S\left(\text { PIVHFOWG }\left(h_{1}, h_{2}, \cdots, h_{n}\right) \leq S\left(\text { PIVHFEOWG }\left(h_{1}, h_{2}, \cdots, h_{n}\right)\right),\right.
$$

The conclusion is that

$$
\operatorname{PIVHFOWG}\left(h_{1}, h_{2}, \cdots, h_{n}\right) \leq \operatorname{PIVHFEOWG}\left(h_{1}, h_{2}, \cdots, h_{n}\right)
$$

If $h_{1}=h_{2}=\cdots=h_{n}$, the equality is established, which completes the proof.

To study the relationship between the PIVHFEOWA operator and the PIVHFEOWG operator, we first introduce Lemma 2 as follows:

Lemma 2. Ref. [20]. Let $a_{j} \geq 0, b_{j} \geq 0, c_{j} \geq 0, d_{j} \geq 0, j=1,2, \cdots, n$ be four sets of non-negative numbers, if $a_{j}-b_{j}-c_{j}-d_{j} \geq 0$ for all $j$, then

$$
\prod_{j=1}^{n} a_{j}-\prod_{j=1}^{n} b_{j}-\prod_{j=1}^{n} c_{j}-\prod_{j=1}^{n} d_{j} \geq 0
$$

Theorem 5. Let $h_{j}(j=1,2, \cdots, n)$ be a set of PIVHFEs and $w=\left(w_{1}, w_{2}, \cdots, w_{n}\right)^{\mathrm{T}}$ be the weighted vector of $h_{j}$, with $w_{j} \geq 0$ and $\sum_{j=1}^{n} w_{j}=1$. As follows:

$$
\operatorname{PIVHFEOWG}\left(h_{1}, h_{2}, \cdots, h_{n}\right) \leq \operatorname{PIVHFEOWA}\left(h_{1}, h_{2}, \cdots, h_{n}\right) \text {. }
$$

If $h_{1}=h_{2}=\cdots=h_{n}$, the equality is established. 
Proof. For any $k=1,2, \cdots, l$, combining the Lemma 1 , we have

$$
\begin{aligned}
& \frac{\prod_{j=1}^{n}\left(1+\gamma_{\sigma(j)}^{(k)-}\right)^{w_{j}}-\prod_{j=1}^{n}\left(1-\gamma_{\sigma(j)}^{(k)-}\right)^{w_{j}}}{\prod_{j=1}^{n}\left(1+\gamma_{\sigma(j)}^{(k)}\right)^{w_{j}}+\prod_{j=1}^{n}\left(1-\gamma_{\sigma(j)}^{(k)}\right)^{w_{j}}}-\frac{2 \prod_{j=1}^{n}\left(\gamma_{\sigma(j)}^{(k)}\right)^{w_{j}}}{\prod_{j=1}^{n}\left(2-\gamma_{\sigma(j)}^{(k)-}\right)^{w_{j}}+\prod_{j=1}^{n}\left(\gamma_{\sigma(j)}^{(k)}\right)^{w_{j}}}= \\
& \frac{\prod_{j=1}^{n}\left(2+\gamma_{\sigma(j)}^{(k)}-\left(\gamma_{\sigma(j)}^{(k)-}\right)^{2}\right)^{w_{j}}-\prod_{j=1}^{n}\left(2-3 \gamma_{\sigma(j)}^{(k)-}+\left(\gamma_{\sigma(j)}^{(k)-}\right)^{2}\right)^{w_{j}}-\prod_{j=1}^{n}\left(\gamma_{\sigma(j)}^{(k)}+\left(\gamma_{\sigma(j)}^{(k)-}\right)^{2}\right)^{w_{j}}-\prod_{j=1}^{n}\left(\sqrt[n]{3} \gamma_{\sigma(j)}^{(k)-}-\sqrt[n]{3}\left(\gamma_{\sigma(j)}^{(k)-}\right)^{2}\right)^{2 w_{j}}}{\left(\prod_{j=1}^{n}\left(1+\gamma_{\sigma(j)}^{(k)-}\right)^{w_{j}}+\prod_{j=1}^{n}\left(1-\gamma_{\sigma(j)}^{(k)}\right)^{w_{j}}\right)\left(\prod_{j=1}^{n}\left(2-\gamma_{\sigma(j)}^{(k)}\right)^{w_{j}}+\prod_{j=1}^{n}\left(\gamma_{\sigma(j)}^{(k)-}\right)^{w_{j}}\right)} .
\end{aligned}
$$

Since $\gamma_{\sigma(j)}^{(k)-} \in[0,1]$ for all $k$ and $j$, then we have

$$
2+\gamma_{\sigma(j)}^{(k)-}-\left(\gamma_{\sigma(j)}^{(k)-}\right)^{2} \geq 0,2-3 \gamma_{\sigma(j)}^{(k)-}+\left(\gamma_{\sigma(j)}^{(k)-}\right)^{2} \geq 0, \gamma_{\sigma(j)}^{(k)-}+\left(\gamma_{\sigma(j)}^{(k)-}\right)^{2} \geq 0, \sqrt[n]{3} \gamma_{\sigma(j)}^{(k)-}-\sqrt[n]{3}\left(\gamma_{\sigma(j)}^{(k)-}\right)^{2} \geq 0, j=1,2, \cdots, n
$$

and pointed out

$$
\left(2+\gamma_{\sigma(j)}^{(k)-}-\left(\gamma_{\sigma(j)}^{(k)-}\right)^{2}\right)-\left(2-3 \gamma_{\sigma(j)}^{(k)-}+\left(\gamma_{\sigma(j)}^{(k)-}\right)^{2}\right)-\left(\gamma_{\sigma(j)}^{(k)-}+\left(\gamma_{\sigma(j)}^{(k)-}\right)^{2}\right)-\left(\sqrt[n]{3} \gamma_{\sigma(j)}^{(k)-}-\sqrt[n]{3}\left(\gamma_{\sigma(j)}^{(k)-}\right)^{2}\right)=\gamma_{\sigma(j)}^{(k)-}(3-\sqrt[n]{3})\left(1-\gamma_{\sigma(j)}^{(k)-}\right) \geq 0
$$

Combining the Lemma 2, we have

$$
\begin{gathered}
\prod_{j=1}^{n}\left(2+\gamma_{\sigma(j)}^{(k)-}-\left(\gamma_{\sigma(j)}^{(k)-}\right)^{2}\right)^{w_{j}}-\prod_{j=1}^{n}\left(2-3 \gamma_{\sigma(j)}^{(k)-}+\left(\gamma_{\sigma(j)}^{(k)-}\right)^{2}\right)^{w_{j}}-\prod_{j=1}^{n}\left(\gamma_{\sigma(j)}^{(k)}+\left(\gamma_{\sigma(j)}^{(k)-}\right)^{2}\right)^{w_{j}}-\prod_{j=1}^{n}\left(\sqrt[n]{3} \gamma_{\sigma(j)}^{(k)-}-\sqrt[n]{3}\left(\gamma_{\sigma(j)}^{(k)-}\right)^{2}\right)^{w_{j}} \geq 0 . \\
\frac{\prod_{j=1}^{n}\left(1+\gamma_{\sigma(j)}^{(k)-}\right)^{w_{j}}-\prod_{j=1}^{n}\left(1-\gamma_{\sigma(j)}^{(k)-}\right)^{w_{j}}}{\prod_{j=1}^{n}\left(1+\gamma_{\sigma(j)}^{(k)-}\right)^{w_{j}}+\prod_{j=1}^{n}\left(1-\gamma_{\sigma(j)}^{(k)-}\right)^{w_{j}}}-\frac{2 \prod_{j=1}^{n}\left(\gamma_{\sigma(j)}^{(k)-}\right)^{w_{j}}}{\prod_{j=1}^{n}\left(2-\gamma_{\sigma(j)}^{(k)-}\right)^{w_{j}}+\prod_{j=1}^{n}\left(\gamma_{\sigma(j)}^{(k)-}\right)^{w_{j}}} \geq 0 .
\end{gathered}
$$

similarly, we have

$$
\frac{\prod_{j=1}^{n}\left(1+\gamma_{\sigma(j)}^{(k)+}\right)^{w_{j}}-\prod_{j=1}^{n}\left(1-\gamma_{\sigma(j)}^{(k)+}\right)^{w_{j}}}{\prod_{j=1}^{n}\left(1+\gamma_{\sigma(j)}^{(k)+}\right)^{w_{j}}+\prod_{j=1}^{n}\left(1-\gamma_{\sigma(j)}^{(k)+}\right)^{w_{j}}}-\frac{2 \prod_{j=1}^{n}\left(\gamma_{\sigma(j)}^{(k)+}\right)^{w_{j}}}{\prod_{j=1}^{n}\left(2-\gamma_{\sigma(j)}^{(k)+}\right)^{w_{j}}+\prod_{j=1}^{n}\left(\gamma_{\sigma(j)}^{(k)+}\right)^{w_{j}}} \geq 0
$$

Therefore, our proof of Theorem 5 is over.

Theorem 6. Let $h_{j}(j=1,2, \cdots, n)$ be a set of PIVHFEs and $w=\left(w_{1}, w_{2}, \cdots, w_{n}\right)^{\mathrm{T}}$ be the weighted vector of $h_{j}$ with $w_{j} \geq 0$ and $\sum_{j=1}^{n} w_{j}=1$. As follows:

$$
\operatorname{PIVHFEOWA}\left(h_{1}, h_{2}, \cdots, h_{n}\right) \leq \operatorname{PIVHFOWA}\left(h_{1}, h_{2}, \cdots, h_{n}\right) .
$$

If $h_{1}=h_{2}=\cdots=h_{n}$, the equality is established.

Proof. For any $k=1,2, \cdots, l$, combining the Lemma 1 , we have

$$
\begin{gathered}
\frac{\prod_{j=1}^{n}\left(1+\gamma_{\sigma(j)}^{(k)-}\right)^{w_{j}}-\prod_{j=1}^{n}\left(1-\gamma_{\sigma(j)}^{(k)}\right)^{w_{j}}}{\prod_{j=1}^{n}\left(1+\gamma_{\sigma(j)}^{(k)-}\right)^{w_{j}}+\prod_{j=1}^{n}\left(1-\gamma_{\sigma(j)}^{(k)-}\right)^{w_{j}}} \\
=1-\frac{2 \prod_{j=1}^{n}\left(1-\gamma_{\sigma(j)}^{(k)-}\right)^{w_{j}}}{\prod_{j=1}^{n}\left(1+\gamma_{\sigma(j)}^{(k)-}\right)^{w_{j}}+\prod_{j=1}^{n}\left(1-\gamma_{\sigma(j)}^{(k)-w_{j}}\right)^{w_{j}}} \leq 1-\frac{2 \prod_{j=1}^{n}\left(1-\gamma_{\sigma(j)}^{(k)-}\right)^{w_{j}}}{2}=1-\prod_{j=1}^{n}\left(1-\gamma_{\sigma(j)}^{(k)-}\right)^{w_{j}},
\end{gathered}
$$

similarly, we have

$$
\frac{\prod_{j=1}^{n}\left(1+\gamma_{\sigma(j)}^{(k)-}\right)^{w_{j}}-\prod_{j=1}^{n}\left(1-\gamma_{\sigma(j)}^{(k)-}\right)^{w_{j}}}{\prod_{j=1}^{n}\left(1+\gamma_{\sigma(j)}^{(k)-}\right)^{w_{j}}+\prod_{j=1}^{n}\left(1-\gamma_{\sigma(j)}^{(k)-}\right)^{w_{j}}} \leq 1-\prod_{j=1}^{n}\left(1-\gamma_{\sigma(j)}^{(k)-}\right)^{w_{j}}
$$

Therefore, our proof of Theorem 6 is over. 
Based on the above Theorems 4-6, we can obtain the size relations between the commonly used information aggregation operators.

Theorem 7. Let $h_{j}(j=1,2, \cdots, n)$ be a set of PIVHFEs and $w=\left(w_{1}, w_{2}, \cdots, w_{n}\right)^{\mathrm{T}}$ be the weighted vector of $h_{j}$, with $w_{j} \geq 0$ and $\sum_{j=1}^{n} w_{j}=1$. As follows:

$\operatorname{PIVHFOWG}\left(h_{1}, h_{2}, \cdots, h_{n}\right) \leq \operatorname{PIVHFEOWG}\left(h_{1}, h_{2}, \cdots, h_{n}\right) \leq \operatorname{PIVHFEOWA}\left(h_{1}, h_{2}, \cdots, h_{n}\right) \leq \operatorname{PIVHFOWA}\left(h_{1}, h_{2}, \cdots, h_{n}\right)$,

with equality holds if and only if $h_{1}=h_{2}=\cdots=h_{n}$.

\section{A Group Decision Making Model Based on the PIVHF Information Aggregation Method}

This section constructs a new MAGDM method based on the proposed information aggregation operators to solve MAGDM problems with probabilistic interval-valued hesitant fuzzy information.

Suppose that $X=\left\{x_{1}, x_{2}, \cdots, x_{m}\right\}$ is a given set of alternatives, $C=\left\{C_{1}, C_{2}, \cdots, C_{n}\right\}$ is attribute set, $w=\left(w_{1}, w_{2}, \cdots, w_{n}\right)^{\mathrm{T}}$ is attribute weighted vector with $w_{j} \geq 0$ and $\sum_{j=1}^{n} w_{j}=1$. A group of experts are invited to make a comprehensive evaluation of the alternatives under the attribute set, in order to select the most comprehensive scheme to make decisions. Secondly, in order to express the decision information provided by the experts more comprehensively and accurately, the probabilistic interval-valued hesitant fuzzy elements $h_{i j}(i=1,2, \cdots, m, j=1,2, \cdots, n)$ will be used to express the evaluation information of the experts under the attribute $x_{i}$ of the scheme $C_{j}$, and then a probabilistic interval-valued hesitant fuzzy decision matrix is constructed.

In the following, the proposed probabilistic interval-valued hesitant fuzzy information aggregation operators will be used to deal with the above MAGDM problems. The specific steps are as follows:

Step 1: Standardized group decision information. If all the attributes are benefit type, the decision matrix A should not be standardized; otherwise, the original matrix is normalized by the following methods:

$$
\widetilde{h}_{i j}=\left\{\begin{array}{c}
h_{i j}, C_{j} \text { is benefit type } \\
h_{i j}^{c}, C_{j} \text { is cost type }
\end{array}, i=1,2, \cdots, m, j=1,2, \cdots, n .\right.
$$

Step 2: Based on the obtained standard group decision matrix $\widetilde{H}=\left(\widetilde{h}_{i j}\right)_{m \times n^{\prime}}$, calculate the comprehensive attribute information $\widetilde{h}_{i}(i=1,2, \cdots, m)$ of each alternative $x_{i}(i=1,2, \cdots, m)$ using the GPIVHFOWA operator:

$$
\begin{gathered}
\widetilde{h}_{i}=\text { GPIVHFOWA }\left(\widetilde{h}_{i 1}, \widetilde{h}_{i 2}, \cdots, \widetilde{h}_{i n}\right)=\bigoplus_{j=1}^{n} w_{j} \widetilde{h}_{i, \sigma(j)} \\
\left.=\left\{\left[f^{-1}\left(\sum_{j=1}^{n} w_{j} f\left(\gamma_{i, \sigma(j)}^{(k)-}\right)\right), f^{-1}\left(\sum_{j=1}^{n} w_{j} f\left(\gamma_{i, \sigma(j)}^{(k)+}\right)\right)\right], \sum_{j=1}^{n} p_{i j}^{(k)}\right) \mid k=1,2, \cdots, l\right\},
\end{gathered}
$$

or using GPIVHFOWG operator:

$$
\begin{gathered}
\widetilde{h}_{i}=\text { GPIVHFOWG }\left(h_{1}, h_{2}, \cdots, h_{n}\right)=\bigotimes_{j=1}^{n} h_{\sigma(j)}^{w_{j}} \\
\left.=\left\{\left[g^{-1}\left(\sum_{j=1}^{n} w_{j} g\left(\gamma_{\sigma(j)}^{(k)-}\right)\right), g^{-1}\left(\sum_{j=1}^{n} w_{j} g\left(\gamma_{\sigma(j)}^{(k)+}\right)\right)\right], \sum_{j=1}^{n} p_{j}^{(k)}\right) \mid k=1,2, \cdots, l\right\},
\end{gathered}
$$

Step 3: According to the calculation formula in Definition 5, the score function value $S\left(\widetilde{h}_{i}\right)$ and the deviation function value $\phi\left(\widetilde{h}_{i}\right)$ of each alternative comprehensive attribute information $\widetilde{h}_{i}(i=1,2, \cdots, m)$ are calculated respectively. 
Step 4: Using the comparison rule of PIVHFE in Definitions 2 and 6, the alternatives are sorted according to their advantages and disadvantages, and the best comprehensive performance scheme is selected.

\section{Illustrative Example}

Nowadays, companies are paying more attention to supply chain management in order to achieve success in the competitions and keep the customers satisfied. The supply chain is a very complex field that involves a large number of participants. The goal of a complete supply chain is to find an optimal solution from the point of view of all participants, which is a rather complicated task. Under the pressure of global competition, enterprises are more inclined to improve the ability of supply chain management to enhance the competitive advantage. The goal of the decision is to minimize the total cost of the supply chain, and how to choose the right supplier is the key to minimize the total cost of the supply chain. Therefore, supplier selection is of prime importance in a supply chain which has a key role in determining the success or failure of a business [33-35].

In order to select suitable suppliers, a dealer's investment department selects five suppliers $X_{i}(i=1,2,3,4,5)$ in accordance with their own needs in the market. The Investment Department invited a group of experts and scholars in related fields to evaluate the five suppliers in the following four indicators, namely $C_{1}$ : Quality, $C_{2}$ : Rate of Return, $C_{3}$ : Delivery ratio, $C_{4}$ : Service and Technology, and index weighted vector is $w=(0.15,0.3,0.2,0.35)^{\mathrm{T}}$.

According to their own professional knowledge and experience, relevant experts give the evaluation information $h_{i j}(i=1,2, \cdots, 5, j=1,2,3,4)$ of each supplier under each index. Furthermore, the probabilistic interval-valued hesitant fuzzy decision matrix $H=\left(h_{i j}\right)_{5 \times 4}$ is shown in Table 1.

Table 1. Probabilistic interval-valued hesitant fuzzy decision matrix $H=\left(h_{i j}\right)_{5 \times 4}$.

\begin{tabular}{|c|c|c|}
\hline & $C_{1}$ & $C_{2}$ \\
\hline$X_{1}$ & $\{([0.6,0.8], 0.5),([0.4,0.6], 0.2),([0.0,0.5], 0.3)\}$ & $\{([0.6,0.8], 0.4),([0.5,0.7], 0.3),([0.2,0.4], 0.3)\}$ \\
\hline$X_{2}$ & $\{([0.5,0.7], 0.6),([0.4,0.6], 0.1),([0.2,0.4], 0.3)\}$ & $\{([0.4,0.6], 0.5),([0.3,0.5], 0.1),([0.1,0.3], 0.4)\}$ \\
\hline$X_{3}$ & $\{([0.7,0.9], 0.2),([0.4,0.6], 0.4),([0.0,0.2], 0.4)\}$ & $\{([0.6,0.8], 0.3),([0.3,0.5], 0.5),([0.2,0.4], 0.2)\}$ \\
\hline$X_{4}$ & $\{([0.4,0.6], 0.6),([0.3,0.5], 0.2),([0.2,0.4], 0.2)\}$ & $\{([0.4,0.6], 0.4),([0.2,0.4], 0.4),([0.0,0.2], 0.2)\}$ \\
\hline \multirow[t]{2}{*}{$X_{5}$} & $\{([0.6,0.8], 0.3),([0.2,0.4], 0.4),([0.1,0.3], 0.3)\}$ & $\{([0.7,0.9], 0.6),([0.6,0.8], 0.2),([0.3,0.5], 0.2)\}$ \\
\hline & $C_{3}$ & $C_{4}$ \\
\hline$X_{1}$ & $\{([0.2,0.4], 0.5),([0.1,0.3], 0.4),([0.0,0.2], 0.1)\}$ & $\{([0.7,0.9], 0.3),([0.4,0.6], 0.3),([0.2,0.4], 0.4)\}$ \\
\hline$X_{2}$ & $\{([0.5,0.7], 0.3),([0.4,0.6], 0.5),([0.0,0.2], 0.2)\}$ & $\{([0.7,0.9], 0.6),([0.5,0.7], 0.3),([0.0,0.2], 0.1)\}$ \\
\hline$X_{3}$ & $\{([0.4,0.6], 0.7),([0.1,0.3], 0.2),([0.0,0.2], 0.1)\}$ & $\{([0.7,0.9], 0.4),([0.2,0.4], 0.5),([0.1,0.3], 0.1)\}$ \\
\hline$X_{4}$ & $\{([0.6,0.8], 0.5),([0.5,0.7], 0.2),([0.3,0.5], 0.3)\}$ & $\{([0.5,0.7], 0.5),([0.3,0.5], 0.3),([0.2,0.4], 0.2)\}$ \\
\hline$X_{5}$ & $\{([0.5,0.7], 0.4),([0.4,0.6], 0.4),([0.1,0.3], 0.2)\}$ & $\{([0.7,0.9], 0.5),([0.4,0.6], 0.1),([0.2,0.4], 0.4)\}$ \\
\hline
\end{tabular}

Step 1: Since the four attribute indexes $C_{j}(j=1,2,3,4)$ are all benefit indicators, it is not necessary to standardize the original probabilistic interval-valued hesitant fuzzy decision matrix.

Step 2: The decision information of each supplier in the attribute index set is integrated by using the GPIVHFOWA operator and the GPIVHFOWG operator (without generality, let additive operator $g(t)=-\ln (t)$. That is, the PIVHFOWA operator and the PIVHFOWG operator, respectively, which compute comprehensive attribute information of each supplier. The calculation results are shown in Tables 2 and 3. 
Table 2. Comprehensive attribute values of each supplier are calculated with the probabilistic interval-valued hesitant fuzzy ordered weighted averaging (PIVHFOWA) operator.

\begin{tabular}{cc}
\hline Suppliers & Comprehensive Decision Attribute Values \\
\hline$X_{1}$ & $\{([0.5845,0.8045], 0.4250),([0.3840,0.5896], 0.3000),([0.1350,0.3816], 0.2750)\}$ \\
$X_{2}$ & $\{([0.5584,0.7774], 0.5000),([0.4104,0.6133], 0.2500),([0.0630,0.2314], 0.2500)\}$ \\
$X_{3}$ & $\{([0.6243,0.8375], 0.4000),([0.2463,0.4487], 0.4000),([0.0986,0.2997], 0.2000)\}$ \\
$X_{4}$ & $\{([0.4809,0.6851], 0.5000),([0.3188,0.5232], 0.2750),([0.1672,0.3693], 0.2250)\}$ \\
$X_{5}$ & $\{([0.6531,0.8618], 0.4500),([0.4453,0.6547], 0.2750),([0.1991,0.4004], 0.2750)\}$ \\
\hline
\end{tabular}

Table 3. Comprehensive attribute values of each supplier are calculated with the probabilistic interval-valued hesitant fuzzy ordered weighted geometric (PIVHFOWG) operator.

\begin{tabular}{cc}
\hline Suppliers & Comprehensive Decision Attribute Values \\
\hline$X_{1}$ & $\{([0.5083,0.7258], 0.4250),([0.3241,0.5471], 0.3000),([0.0000,0.3601], 0.2750)\}$ \\
$X_{2}$ & $\{([0.5261,0.7298], 0.5000),([0.3967,0.5996], 0.2500),([0.0000,0.2259], 0.2500)\}$ \\
$X_{3}$ & $\{([0.5976,0.8011], 0.4000),([0.2182,0.4291], 0.4000),([0.0000,0.2838], 0.2000)\}$ \\
$X_{4}$ & $\{([0.4690,0.6708], 0.5000),([0.2942,0.5002], 0.2750),([0.0000,0.3397], 0.2250)\}$ \\
$X_{5}$ & $\{([0.6395,0.8409], 0.4500),([0.4071,0.6155], 0.2750),([0.1772,0.3867], 0.2750)\}$ \\
\hline
\end{tabular}

Step 3: According to the Definition 5, the score function value of each supplier's comprehensive attribute information $h_{i}(i=1,2,3,4,5)$ in Table 4 is calculated.

Table 4. Score functions of each supplier are calculated with different operators.

\begin{tabular}{cccccc}
\hline Operators & $\boldsymbol{X}_{\mathbf{1}}$ & $\boldsymbol{X}_{\mathbf{2}}$ & $\boldsymbol{X}_{\mathbf{3}}$ & $\boldsymbol{X}_{\mathbf{4}}$ & $\boldsymbol{X}_{\mathbf{5}}$ \\
\hline PIVHFOWA & {$[0.1336,0.2079]$} & {$[0.1325,0.1999]$} & {$[0.1227,0.1915]$} & {$[0.1219,0.1898]$} & {$[0.1570,0.2260]$} \\
PIVHFOWG & {$[0.1044,0.1905]$} & {$[0.1207,0.1904]$} & {$[0.1088,0.1829]$} & {$[0.1051,0.1831]$} & {$[0.1495,0.2180]$} \\
PIVHFEOWA & {$[0.1313,0.2062]$} & {$[0.1312,0.1989]$} & {$[0.1215,0.1905]$} & {$[0.1208,0.1888]$} & {$[0.1560,0.2251]$} \\
PIVHFEOWG & {$[0.1074,0.1938]$} & {$[0.1218,0.1917]$} & {$[0.1098,0.1842]$} & {$[0.1057,0.1841]$} & {$[0.1506,0.2192]$} \\
IVHFOWA & {$[0.3678,0.5919]$} & {$[0.3439,0.5407]$} & {$[0.3231,0.5286]$} & {$[0.3223,0.5259]$} & {$[0.4325,0.6390]$} \\
IVHFOWG & {$[0.2775,0.5443]$} & {$[0.3076,0.5184]$} & {$[0.2719,0.5047]$} & {$[0.2544,0.5036]$} & {$[0.4079,0.6144]$} \\
\hline
\end{tabular}

Step 4: The five suppliers are sorted according to the value of the score function. The greater the score function value is, the better the supplier's comprehensive condition is. The results of the specific sorting are shown in Table 5. By using the PIVHFOWA operator and the PIVHFOWG operator, the suppliers with optimal conditions are all $X_{5}$.

Table 5. Ranking results of the five suppliers with different operators.

\begin{tabular}{cl}
\hline Operators & Ranking Results \\
\hline PIVHFOWA operator & $X_{5} \succ X_{1} \succ X_{2} \succ X_{3} \succ X_{4}$ \\
PIVHFOWG operator & $X_{5} \succ X_{2} \succ X_{1} \succ X_{3} \succ X_{4}$ \\
PIVHFEOWA operator & $X_{5} \succ X_{1} \succ X_{2} \succ X_{3} \succ X_{4}$ \\
PIVHFEOWG operator & $X_{5} \succ X_{2} \succ X_{1} \succ X_{3} \succ X_{4}$ \\
IVHFOWA operator & $X_{5} \succ X_{1} \succ X_{2} \succ X_{3} \succ X_{4}$ \\
IVHFOWG operator & $X_{5} \succ X_{2} \succ X_{1} \succ X_{3} \succ X_{4}$ \\
\hline
\end{tabular}

In order to explore the similarities and differences of decision results, we will use the PIVHFEOWA operator and the PIVHFEOWG operator to deal with the above problems and get the score function of each vendor's attribute value, the calculation results are shown in Table 4 . Then, according to the value of the score function, five suppliers are sorted, and the results are shown in Table 5. As shown in Table 5, after using the PIVHFEOWA operator and the PIVHFEOWG operator for information 
aggregation, the suppliers with the best comprehensive performance are $X_{5}$. Therefore, the results obtained by using the four operators proposed in this paper are the same, which shows that the generalized probabilistic hesitant fuzzy information aggregation operators proposed in this paper has inherent consistency.

In order to illustrate the rationality and reliability of multi attribute group decision making method in this paper, the interval-valued hesitant fuzzy ordered weighted averaging (IVHFOWA) operator and interval hesitant fuzzy ordered weighted geometric (IVHFOWG) operator (Equations (27) and (28)) are compared and analyzed in [28]. Using the method of [28] to deal with the above supplier selection problem, the steps are as follows:

- Step 1': Same as step 1.

- Step 2': Based on the original decision matrix $H=\left(h_{i j}\right)_{5 \times 4^{\prime}}$, the comprehensive attribute values $h_{i}(i=1,2,3,4,5)$ of each supplier are calculated by using Equations (27) and (28). Considering the limitation of the length of the paper and the elements in $h_{i}$ can reach up to 81 , then we are not list the comprehensive attribute values $h_{1}$ in details.

- Step 3': Compute the score functions (Definition 3) $S\left(h_{i}\right)(i=1,2,3,4,5)$ of the comprehensive IVHFEs, which are shown in Table 4.

- Step $4^{\prime}$ : According to the value of the score function, five suppliers are sorted, and the results are shown in Table 5 . The calculation results show that the supplier with the best comprehensive performance is $X_{5}$.

From the above analysis, we can see that the proposed method in this article has the same result with that of Chen et al. [28]. That is, the optimal selection is $X_{5}$, which illustrates the MAGDM method in this paper is reasonable. Although the ranking of the five selections is slightly different, the worst selection is $X_{4}$ with different operators. Besides, the MAGDM model in this paper is based on a variety of aggregation operators, which can reflect different decision results. Using the MAGDM model in this paper, decision makers can choose different information aggregation operators with their subjective attitudes. Therefore, the method proposed in this paper is convenient. Furthermore, compared with the method developed by Chen et al. [28], we find that our proposed method has some advantages:

(1) Probabilistic interval-valued hesitant fuzzy elements can not only represent all possible decision information, but also reflect the importance of each decision information.

(2) Comparing the numbers of interval-valued hesitant fuzzy elements, it is found that the decision making process of the information aggregation proposed by our method is more simplified and convenient than that of Chen et al. [28].

(3) According the overall results of PIVHFEs derived by the PIVHFOWA operator, the PIVHFOWG operator, the PIVHFEOWA operator and the PIVHFEOWG operator, it is found that the relationship among these probabilistic interval-valued hesitant fuzzy aggregation operators are consistent with the Theorem 7.

In fact, the PIVHFOWA operator and the PIVHFEOWA operator pay more attention to the influence of all attribute information, while the PIVHFOWG operator and the PIVHFEOWG operator reflect the importance of single attribute information. Decision makers can choose aggregation operators according to their subjective attitudes.

\section{Conclusions}

The aggregation operators proposed in this paper have shown some advantages over the IVHFOWA operator and the IVHFOWG operator in reducing the loss of decision information. In this paper, the main contribution is to put forward a method of probabilistic interval-valued hesitant fuzzy information aggregation which combines the idea of Archimedes norm. Using the novel method, the problem of information loss can be solved more effectively in practical MAGDM problems. 
In addition, the integration of probability makes decision results more accurate. Taking supplier selection as an example, this paper illustrates the feasibility and effectiveness of the proposed method.

This paper only considers the equal weight of experts in the process of group decision making, but needs further study for unequal weights. In addition, there are four different forms in Archimedes norm, such as Algebraic, Einstein, Hamacher and Frank t-conorms and t-norms. But only two of them are selected in this paper, and the other two forms can be discussed in the future study.

Author Contributions: W.W. proposed the overall idea, algorithm and the detailed writing, including compute and analysis; F.J. discussed the algorithm; Y.L., Z.N. and X.Z. reviewed and checked the language of the paper.

Funding: This research is funded by the National Social Science Foundation of China (No. 16BJY132), the Program of National Natural Science Foundation of China (No. 91546108), the Key Project of Natural Science Foundation of China (No. 71490725), the Philosophy and Social Science Programming Item of Anhui Province (AHSKY2015D68), the scholarship from China Scholarship Council (No. 201706690001).

Acknowledgments: This paper is supported by the National Social Science Foundation of China (No. 16BJY132), the Program of National Natural Science Foundation of China (No. 91546108), the Key Project of Natural Science Foundation of China (No. 71490725), the Philosophy and Social Science Programming Item of Anhui Province (AHSKY2015D68), the scholarship from China Scholarship Council (No. 201706690001). The authors are very thankful to the editors and referees for their valuable comments and suggestions for improving the paper.

Conflicts of Interest: The authors declare no conflict of interest.

\section{References}

1. Zadeh, L.A. Fuzzy sets. Inf. Control 1965, 8, 338-353. [CrossRef]

2. Atanassov, K. Intuitionistic fuzzy sets. Fuzzy Sets Syst. 1986, 20, 87-96. [CrossRef]

3. Torra, V. Hesitant fuzzy sets. Int. J. Intell. Syst. 2010, 25, 529-539. [CrossRef]

4. Atanassov, K. Interval valued intuitionistic fuzzy sets. Fuzzy Sets Syst. 1989, 31, 343-349. [CrossRef]

5. Garg, H. A new generalized improved score function of interval-valued intuitionistic fuzzy sets and applications in expert systems. Appl. Soft Comput. 2016, 38, 988-999. [CrossRef]

6. Zhang, F.W.; Ge, Y.E.; Garg, H.; Luo, L.H. Commentary on "A new generalized improved score function of interval-valued intuitionistic fuzzy sets and applications in expert systems". Appl. Soft Comput. 2017, 52, 48-52. [CrossRef]

7. Wei, C.P.; Zhang, Y.Z. Entropy Measures for Interval-Valued Intuitionistic Fuzzy Sets and Their Application in Group Decision-Making. Math. Probl. Eng. 2015, 2015, 563745. [CrossRef]

8. Nguyen, H. A new interval-valued knowledge measure for interval-valued intuitionistic fuzzy sets and application in decision making. Expert Syst. Appl. 2016, 56, 143-155. [CrossRef]

9. Chen, X.H.; Zhao, C.C.; Yang, L. A group decision-making model based on interval-valued intuitionistic fuzzy numbers and its application on social network. Syst. Eng. Theory Pract. 2017, 37, 1842-1852.

10. Farhadinia, B. A Multiple Criteria Decision Making Model with Entropy Weight in an Interval-Transformed Hesitant Fuzzy Environment. Cogn. Comput. 2017, 9, 513-525. [CrossRef]

11. Chen, S.M.; Huang, Z.C. Multi attribute Decision Making Based on Interval-Valued Intuitionistic Fuzzy Values and Particle Swarm Optimization Techniques. Inf. Sci. 2017, 397, 206-218. [CrossRef]

12. Chen, S.W.; Cai, L.N. Interval-valued Hesitant Fuzzy Sets. Fuzzy Syst. Math. 2013, 27, 38-44.

13. Jin, F.F.; Ni, Z.W.; Chen, H.Y.; Li, Y.; Zhou, L. Multiple attribute group decision making based on interval-valued hesitant fuzzy information measures. Comput. Ind. Eng. 2016, 101, 103-115. [CrossRef]

14. Liu, J.; Zhou, N.; Zhuang, L.H.; Li, N.; Jin, F.F. Interval-Valued Hesitant Fuzzy Multi-attribute Group Decision Making Based on Improved Hamacher Aggregation Operators and Continuous Entropy. Math. Probl. Eng. 2017, 2017, 2931482. [CrossRef]

15. Lan, J.B.; Zheng, Z.Y.; Jin, R.F.; Hu, M.M. Multiple attribute decision making under interval-valued hesitant fuzzy sets: An priority degrees approach. In Proceedings of the International Conference on Progress in Informatics and Computing (PIC), Shanghai, China, 23-25 December 2016; pp. 97-101.

16. Xian, S.D.; Dong, Y.F.; Yin, Y.B. Interval-valued intuitionistic fuzzy combined weighted averaging operator for group decision making. J. Oper. Res. Soc. 2017, 68, 895-905. [CrossRef] 
17. Gitinavard, H.; Mousavi, S.M.; Vahdani, B. A new multi-criteria weighting and ranking model for group decision-making analysis based on interval-valued hesitant fuzzy sets to selection problems. Neural Comput. Appl. 2016, 27, 1593-1605. [CrossRef]

18. Shang, Z.W.; Guo, Y.H.; Zou, J.G.; Yan, J.Q. Interval number group decision making method with unknown objective weights of attributes and decision makers. Comput. Eng. Appl. 2017, 53, 227-232.

19. Li, L.; Wang, Y.Q. A New Method Determining the Weights of Experts in Multiple-Attribute Group Decision-Making. Stat. Decis. 2017, 2, 14-18.

20. Jin, F.F.; Ni, Z.W.; Chen, H.Y. Interval-valued hesitant fuzzy Einstein prioritized aggregation operators and their applications to multi-attribute group decision making. Soft Comput. 2016, 20, 1863-1878. [CrossRef]

21. Zhang, S.; Xu, Z.S.; He, Y. Operations and integrations of probabilistic hesitant fuzzy information in decision making. Inf. Fusion 2017, 38, 1-11. [CrossRef]

22. Wang, Z.X.; Li, J. Correlation Coefficients of Probabilistic Hesitant Fuzzy Elements and Their Applications to Evaluation of the Alternatives. Symmetry 2017, 9, 259. [CrossRef]

23. Zhai, Y.L.; Xu, Z.S.; Liao, H.C. Measures of probabilistic interval-valued intuitionistic hesitant fuzzy sets and the application in reducing excessive medical examinations. IEEE Trans. Fuzzy Syst. 2018, 26, 1651-1670. [CrossRef]

24. Liu, H.B.; Jiang, L.; Xu, Z.S. Entropy Measures of Probabilistic Linguistic Term Sets. Int. J. Comput. Intell. Syst. 2018, 11, 45-57. [CrossRef]

25. Farhadinia, B.; Xu, Z.S. Ordered Weighted Hesitant Fuzzy Information Fusion-Based Approach to Multiple Attribute Decision Making with Probabilistic Linguistic Term Sets. Fundam. Inform. 2017, 159, 361-383. [CrossRef]

26. Li, J.; Wang, Z.X. Consensus Building for Probabilistic Hesitant Fuzzy Preference Relations with Expected Additive Consistency. Int. J. Fuzzy Syst. 2018, 20, 1495-1510. [CrossRef]

27. Zhou, W.; Xu, Z.S. Group consistency and group decision making under uncertain probabilistic hesitant fuzzy preference environment. Inf. Sci. 2017, 414, 276-288. [CrossRef]

28. Chen, N.; Xu, Z.S.; Xia, M.M. Interval-valued hesitant preference relations and their applications to group decision making. Knowl. Based Syst. 2013, 37, 528-540. [CrossRef]

29. Chen, N.; Xu, Z.S.; Xia, M.M. Correlation coefficients of hesitant fuzzy sets and their applications to clustering analysis. Appl. Math. Model. 2013, 37, 2197-2211. [CrossRef]

30. Liu, P.D. The Aggregation Operators Based on Archimedean t-Conorm and t-Norm for Single-Valued Neutrosophic Numbers and their Application to Decision Making. Int. J. Fuzzy Syst. 2016, 18, 849-863. [CrossRef]

31. Liu, P.D.; Chen, S.M. Heronian aggregation operators of intuitionistic fuzzy numbers based on the Archimedean t-norm and t-conorm. In Proceedings of the International Conference on Machine Learning and Cybernetics (ICMLC), Jeju, South Korea, 10-13 July 2017; pp. 686-691.

32. Xia, M.M.; Xu, Z.S. Hesitant fuzzy information aggregation in decision making. Int. J. Approx. Reason. 2011, 52, 395-407. [CrossRef]

33. Kriheli, B.; Levner, E. Entropy-Based Algorithm for Supply-Chain Complexity Assessment. Algorithms 2018, 11, 35. [CrossRef]

34. Shao, M.H.; Song, Y.; Wu, B.; Chang, Y.J. The Supplier Selection of the Marine Rescue Equipment Based on the Analytic Hierarchy Process (AHP)-Limited Diversity Factors Method. Algorithms 2018, 11, 63. [CrossRef]

35. Afzali, A.; Rafsanjani, M.K.; Saeid, A.B. A Fuzzy Multi-objective Linear Programming Model Based on Interval-valued Intuitionistic Fuzzy Sets for Supplier Selection. Int. J. Fuzzy Syst. 2016, 18, 864-874. [CrossRef]

(C) 2018 by the authors. Licensee MDPI, Basel, Switzerland. This article is an open access article distributed under the terms and conditions of the Creative Commons Attribution (CC BY) license (http:/ / creativecommons.org/licenses/by/4.0/). 